\title{
Características salivales y estado sistémico de sujetos con xerostomía
}

\section{Salivary characteristics and systemic status of subjects with xerostomia}

\author{
Juan Pablo Aitken-Saavedra ${ }^{1-4}$, Cristobal Olid², Alejandro Escobar ${ }^{3}$, Yoshua Parry², \\ Karine Duarte da Silva ${ }^{4}$, Irene Morales-Bozo ${ }^{3^{*}}$
}

\author{
1. Universidad de Chile, Departamento de Patología \\ y Medicina Oral \\ 2. Universidad de Chile, Facultad de Odontología \\ 3. Universidad de Chile, Instituto de Investigación en \\ Ciencias Odontológicas \\ 4. Post Graduate Program in Dentistry, School of \\ Dentistry, Federal University of Pelotas, Pelotas-RS, \\ Brazil \\ * Correspondence: Irene Morales-Bozo \\ Address: Sergio Livingstone Pohlhammer 943 \\ Independencia. Santiago, Chile \\ E-Mail: imorales@u.uchile.cl \\ Trabajo recibido el 23/02/2017. Aprobado para su \\ publicación el 28/05/2017
}

\section{RESUMEN}

Xerostomía o sensación de boca es una afección que afecta severamente la calidad de vida de quienes la padecen. Si bien se relaciona con la reducción del flujo salival (hiposalivación), existe evidencia contradictoria y se sugiere evaluar características cualitativas salivales y estado sistémico de los afectados para comprender su etiología y mejorar terapias asociadas. El objetivo de este estudio fue comparar $\mathrm{pH}$ y concentración de proteínas en saliva y estado sistémico entre sujetos xerostómicos con y sin hiposialia.

Se midió $\mathrm{pH}$, concentración de proteínas salivales y se consignaron antecedentes sistémicos y uso de fármacos en 27 individuos xerostómicos, que fueron divididos en hiposiálicos y no hiposiálicos. Se compararon las variables mencionadas usando test no paramétrico de Mann-Whitney y test Chi-Cuadrado.

Se aceptaron diferencias estadísticamente significativas con error alfa igual o menor a $5 \%$. Sujetos xerostómicos con hiposialia presentaron mayor prevalencia de Artritis Reumatoide y Síndrome de Sjögren en comparación con xerostómicos sin hiposialia. No se encontraron diferencias estadísticamente significativas en relación a $\mathrm{pH}$, concentración de proteínas y uso de medicamentos, variables que no influirían en el padecimiento de xerostomía, independiente de la existencia de hiposialia. Sujetos con xerostomía e hiposialia presentan mayor frecuencia de Artritis Reumatoide y Síndrome de Sjögren.

\section{PALABRAS CLAVE}

Xerostomía, Hiposialia, Síndrome de Sjögren

Rev. Clin. Periodoncia Implantol. Rehabil. Oral Vol. 10(2); 118-120, 2017.

\begin{abstract}
Xerostomia or mouth feeling is a condition that severely affects the quality of life of thosewho suffer from it. Although it is related to the reduction of salivary flow (hyposalivation), there is contradictory evidence and it is suggested to evaluate qualitative salivarycharacteristics and systemic state of those affected in order to understand its etiology and improve associated terapies. The objective of this study was to compare saliva, $\mathrm{pH}$ and protein concentration and systemic status among xerostomic subjects with and without hyposialia. Were measured $\mathrm{PH}$ and protein concentration and were recorded systemic antecedents and drug use in 27 xerostomic individuals, who were divided into hyposalic and nonhyposalic individuals. The mentioned variables were compared using non-parametric Mann-Whitney test and Chi-Square test. Statistically significant differences were accepted with alpha error equal to or less than $0.05 \%$. Xerostomic subjects with hyposialia presented higher prevalence of Rheumatoid Arthritis and Sjögren's Syndrome compared to xerostomics without hyposialia. No statistically significant differences were found in relation to $\mathrm{pH}$, protein concentration and drug use, variables that would not influence xerostomia, independent of hyposialia. Subjects with xerostomia with hyposialia present a higher frequency of Rheumatoid Arthritis and Sjögren's Syndrome.

KEYWORDS

Hyposalivation, Xerostomia, Sjogren`s Syndrome
\end{abstract}

Rev. Clin. Periodoncia Implantol. Rehabil. Oral Vol. 10(2); 118-120, 2017. 


\section{INTRODUCCION}

Xerostomía o sensación de boca seca es una afección compleja que afecta variadas funciones estomatológicas y va en detrimento de la calidad de vida de los individuos que la padecen por lo que se ha convertido en una causa frecuente de consulta odontológica en la actualidad (1). Este síntoma, se ha relacionado con frecuencia con la reducción del flujo salival (hiposalivación) que cuando alcanza una producción menor a $0,2 \mathrm{ml} / \mathrm{min}$ se denomina hiposialia, relacionándose fuertemente con mayor número de lesiones orales, disgeusia, disfagia, disartria, caries cervicales y mayor susceptibilidad a infecciones producidas por hongos, entre otras afecciones ${ }^{(2-4)}$. Sin embargo, existe evidencia que establece que no necesariamente la xerostomía está acompañada de una disminución del flujo salival y sugiere estudiar más profundamente, características cualitativas salivales, así como a la condición sistémica del afectado para comprender mejor la etiología del síntoma y aportar en la búsqueda de una terapia que resuelva o mejore este padecimiento.

La evidencia respecto de la asociación de xerostomía con características cualitativas salivales como variaciones en el pH o en la composición de proteínas salivales, todavía es escasa y se sugieren estudios complementarios. El estado fisiológico de los sujetos afectados como el envejecimiento o pertenecer al género femenino, así como el consumo de fármacos asociados a ciertas enfermedades crónicas (antidepresivos tricíclicos, ansiolíticos, antihipertensivos, entre otros), también podrán estar determinando el padecimiento del síntoma ${ }^{(5,6)}$

Actualmente, las terapias destinadas a tratar la xerostomía y mejorar la calidad de vida de los sujetos afectados, se enfocan a aumentar el flujo salival sin evaluar previamente, si el padecimiento del síntoma está determinado exclusivamente por la disminución de la cantidad de saliva producida(7). Es por esto que se hace preciso evaluar y comparar no sólo las características cuantitativas, sino también algunas cualitativas como $\mathrm{pH}$ y niveles de proteínas salivales, así como características sistémicas y consumo de fármacos entre sujetos que presentan xerostomía con y sin hiposialia. Los resultados de este estudio, podrían ampliar el conocimiento acerca de la etiología y el papel de las características salivales y estado sistémico de los sujetos afectados en la determinación del padecimiento de xerostomía e hiposialia, pudiendo ser un aporte en la indicación de terapias asociadas.

\section{MATERIALES Y METODOS}

Tipo de estudio. Transversal descriptivo. Se incluyeron 29 sujetos, reclutados en el Servicio de Diagnóstico de la Facultad de Odontología de la Universidad de Chile, mayores de 18 años de ambos géneros con diagnóstico de xerostomía de cualquier origen según la encuesta de $\mathrm{Fox}^{(8)}$. Se excluyeron sujetos con trastornos motores a nivel bucal, deterioro cognitivo severo y aquellos con actual tratamiento de estimulación salival. El estudio fue aprobado por el Comité de Ética de la Facultad de Odontología de la Universidad de Chile y fue realizado conforme a la declaración de Helsinki(9). Un equipo de odontólogos calibrados, realizaron la anamnesis y el examen clínico al grupo de estudio. Se aplicó la encuesta de FOX para identificar a los individuos con xerostamía y para determinar la severidad de ésta. Se consignaron las enfermedades sistémicas que padecía el sujeto, junto con los datos respecto de la medicación asociada en cada caso.

Determinación de velocidad de flujo salival (VFS). Se realizó el test de saliva total no estimulada ${ }^{(10)}$. La medición se realizó entre las 8 y 11 horas A.M. Se solicitó a los individuos depositar saliva en tubos Falcon ${ }^{\circledR}$ de $50 \mathrm{ml}$, estériles y previamente pesados durante cinco minutos. La saliva recolectada, fue pesada y expresada en $\mathrm{ml} / \mathrm{min}$, asumiendo un valor de densidad igual a 1 para la saliva. Se estableció padecimiento de hiposialia como tasas de flujo salival menor o igual a $0,2 \mathrm{ml} / \mathrm{min}^{(11)}$.

Determinación del pH salival. Para la medición del valor del $\mathrm{pH}$ de las muestras salivales, se empleó un pH-metro digital (Modelo PL-600 EZDOOMEGA que cumple la norma ISO-9001), que de forma automatizada ofrece el valor del $\mathrm{pH}$ de forma digital con 2 decimales. Todas las mediciones se realizaron por el mismo operador siguiendo las instrucciones del fabricante.

Determinación de la concentración total de proteínas. Se utilizó el kit para cuantificación de proteínas Protein Assay BioRad (Richmond CA, USA) y estándares de albúmina bovina (BSA, Sigma). Las muestras de saliva a analizar se procesaron en duplicado y se realizó la lectura de absorbancia en un espectrofotómetro a $595 \mathrm{~nm}$. Se confeccionó una curva de calibración y se estimó la concentración de proteínas totales en ug/mL según las instrucciones del fabricante.

Análisis estadístico. Se utilizó el test no paramétrico de Mann-Whitney para la comparación de $\mathrm{pH}$ y concentración de proteínas y el test Chi-Cuadrado para comparar prevalencia de enfermedades sistémicas y consumo de fármacos entre sujetos xerostómicos con y sin hiposialia. Se aceptaron diferencias estadísticamente significativas con un error alfa igual o menor a $0,05 \%$ y un intervalo de confianza del 95\%. Los análisis se realizaron usando el programa Excel y Graph Pad.

\section{RESULTADOS}

Análisis de datos demográficos El total de la muestra comprendió a 29 pacientes con xerostomía, de los cuales, 2 eran asiálicos (sin producción de saliva que pudiese ser cuantificada). De los 27 sujetos a los que se pudo realizar los análisis, 21 (77\%) eran mujeres. La media de edad fue de 57,1 años (DS 20,5 años), fluctuando en un rango de entre 21 y 87 años. El $91 \%$ de los sujetos con hiposialia y el 68,8 de los sin hiposialia, eran mujeres. (Tabla 1)

Tabla 1. Caracterización de la muestra.

\begin{tabular}{|c|c|c|c|}
\hline & $\begin{array}{c}\text { Hiposiálicos } \\
\mathbf{n}(\%)\end{array}$ & $\begin{array}{c}\text { No hiposiálicos } \\
\mathbf{n}(\%)\end{array}$ & Total $\mathbf{n}(\%)$ \\
\hline Hombres $\mathrm{n}(\%)$ & $1(9 \%)$ & $5(31,2 \%)$ & $6(22,2 \%)$ \\
Mujeres $\mathrm{n}(\%)$ & $10(91 \%)$ & $11(68,8 \%)$ & $21(77,7 \%)$ \\
Edad (años / DS) & $60,5(17,9 \%)$ & $54,8(21,2 \%)$ & $57,1(20,5 \%)$ \\
Total $\mathrm{n}(\%)$ & $11(40,74 \%)$ & $16(59,26 \%)$ & $27(100 \%)$
\end{tabular}

Determinación de velocidad del flujo salival (VFS) en sujetos xerostómicos con y sin hiposialia. La mediana de VFS de hiposiálicos y no hiposiálicos fue de $0,1 \mathrm{ml} / \mathrm{min}$ y $0,45 \mathrm{ml} / \mathrm{min}$ respectivamente.

Comparación del $\mathrm{pH}$ salival entre sujetos xerostómicos con y sin hiposialia. La mediana de $\mathrm{pH}$ fue de 8,0 y 7,95 en sujetos xerostómicos con y sin hiposialia respectivamente. No se observó diferencia estadísticamente significativa al comparar ambos grupos ( $p>0,05)$.

Comparación de concentración de proteínas salivales entre sujetos xerostómicos con y sin hiposialia. La mediana para la concentración de proteínas salivales de individuos xerostómicos con y sin hiposialia fue de 0,12 y $0,13 \mathrm{ug} / \mathrm{mL}$ respectivamente. No se observó diferencia estadísticamente significativa al comparar ambos grupos $(p>0,05)$.

Comparación de enfermedades sistémicas entre sujetos xerostómicos hiposiálicos y no hiposiálicos. Se observó diferencia estadísticamente significativa al comparar prevalencia de Artritis Reumatoide y Síndrome de Sjögren entre sujetos xerostómicos hiposiálicos con respecto a los xerostómicos no hiposiálicos $(p<0,05)$. (Figura 1)

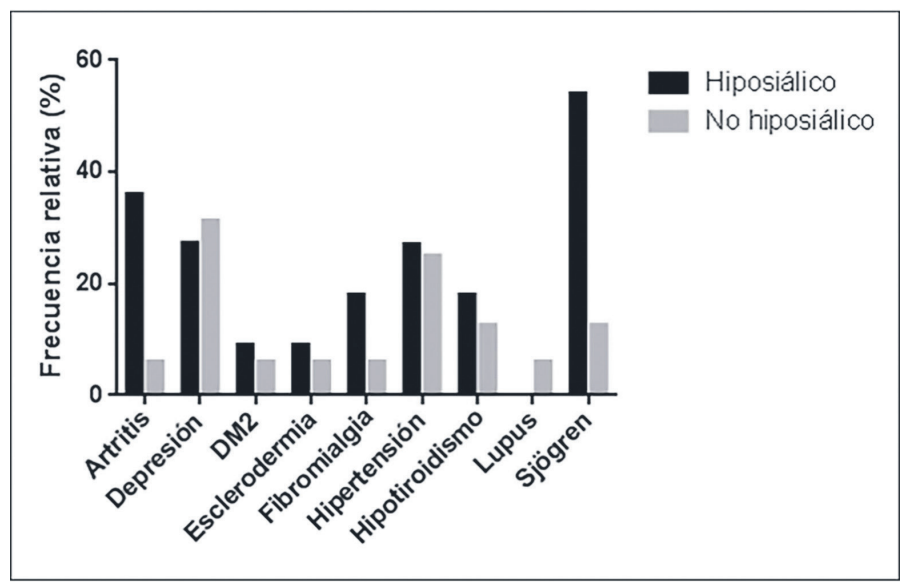

Se destaca una diferencia estadísticamente significativa al comparar prevalencia de Artritis Reumatoide (Artritis) y Síndrome de Sjögren (Sjögren) entre sujetos xerostómicos hiposiálicos (Hiposiálico) y xerostómicos no hiposiálicos (No hiposiálico) $(p<0,05)$

Figura 1. Frecuencia de enfermedades sistémicas en sujetos xerostómicos hiposiálicos y no hiposiálicos.

Comparación del número de medicamentos consumidos entre sujetos xerostómicoshiposiálicos y no hiposiálicos. No se observó diferencia estadísticamente significativa al comparar frecuencia de consumo de medicamentos entre sujetos xerostómaticos con y sin hiposialia $(p>0,05)$.

\section{DISCUSION}

El objetivo de este estudio fue comparar velocidad de flujo salival, $\mathrm{pH}$, concentración de proteínas salivales y prevalencia de enfermedades sistémicas entre sujetos con xerostomía con y sin hiposialia, para así, ampliar el conocimiento respecto de la etiología de la xerostomía y aportar conocimiento que permita mejorar las terapias destinadas a mejorar la calidad de vida de los afectados. Si bien los participantes de este estudio eran todos xerostómicos, sólo un 40,24\% tenían hiposialia. Estos resultados concuerdan con reportes 
que establecen que la xerostomía no se relaciona necesariamente con la disminución de la velocidad del flujo salival ${ }^{(4,12)}$ pero es contradictoria con reportes que relacionan ambos fenómenos ${ }^{(2)}$

En relación a la edad, no hubo diferencias estadísticamente significativas entre los individuos con y sin hiposialia, por lo que podemos sugerir que al menos en nuestro estudio, la edad no es un factor determinante en la disminución del flujo salival. Sin embargo, debe considerarse que existe evidencia que establece que a mayor edad, hay un deterioro del parénquima glandular, el que se reemplaza por tejido conectivo disminuyendo el flujo salival(13). El porcentaje de mujeres xerostómicas en nuestro estudio, correspondió al $77 \%$, lo que podría relacionarse, si asociamos el síntoma con la disminución del flujo salival, al menor tamaño y capacidad funcional de las glándulas salivales que presentan las mujeres en comparación con los hombre ${ }^{(14)}$. Estos datos además, sugieren establecer parámetros diferenciados para identificar hiposialia en ambos generos. Un estudio previo, había establecido que la xerostomía sin hiposialia, se asocia al género femenino y que la depresión, estrés, ansiedad e ingesta de antihipertensivos, relacionados más con mujeres, pueden ser factores determinen el padecimiento del síntoma ${ }^{(15)}$. Nuestros resultados sugieren que al menos en mujeres, la xerostomía no se relaciona necesariamente a la hiposialia.

Al comparar pH salival entre xerostómicos hiposiálicos y no hiposiálicos, las diferencias no fueron estadísticamente significativas, lo que es coincidente con un estudio que establece que no existen variaciones de $\mathrm{pH}$ a medida que varía el flujo salival(16), pero difiere con lo relatado por otros autores, que establecen que el $\mathrm{pH}$ se torna más alcalino a mayor flujo, pudiendo variar de 6,6 (saliva total no estimulada) a 7,4 (saliva estimulada) debido a un sistema de amortiguación de carbonato y fosfato ${ }^{(12)}$. Con respecto a la relación entre xerostomía y $\mathrm{pH}$ salival, existen estudios que sugieren asociación pero a la vez también indican una multifactorialidad en la etiología del síntoma ${ }^{(17)}$. Un estudio realizado en sujetos que manifestaron tener xerostomía luego de una ser irradiados en la zona de cabeza y cuello, señala una leve disminución de $\mathrm{pH}$ salival después de la terapia, lo que podría sugerir una posible relación entre acidez salival y sensación de boca seca, sin embargo, el análisis no es conclusivo al respecto ${ }^{(18)}$. En nuestro estudio al menos, valores de $\mathrm{pH}$ salival no serían un factor determinante en el padecimiento de xerostomía.

Al comparar los niveles de proteínas salivales entre hiposiálicos y no hiposiálicos, no se observaron diferencias estadísticamente significativas. Sin embargo, los niveles destacados, estuvieron por debajo de los reportdos por la literatura ${ }^{(19)}$. Esto podría deberse a que nuestro estudio consideró solamente medición de saliva no estimulada y no se evaluaron variables como enfernedad periodontal, donde el exudado de proteínas por la vía del fluido crevicular, dependiente del grado de inflamación, podría influenciar la medición de proteínas totales en saliva ${ }^{(13,20)}$

Al comparar frecuencia de enfermedades sistémicas entre ambos grupos de estudio, se observó que Artritis Reumatoide y Síndrome de Sjögren, ambas de etiología autoinmune,fueron más prevalentes en el grupo de xerostómicos con hiposialia. Existe evidencia, que indica que enfermedades autoinmunes como las señaladas, alteran el parénquima glandular salival, lo que se expresaría como hiposalivación ${ }^{(21,22)}$. Otras enfermedades como depresión e hipertensión se presentaron en alto número en sujetos de ambos grupos. La presencia de enfermedades sistémicas como las autoinmunes descritas y otras como diabetes mellitus tipo $2^{(23)}$, también podrían asociarse a un menor flujo salival, pero además, con la alteración de algunas características salivales, especialmente con la concentración de proteínas en saliva. Si bien a este respecto, no fue posible detectar diferencias entre los niveles de proteínas salivales entre sujetos con y sin hiposialia, tanto la Artritis Reumatoide como el Síndrome de Sjögren, podrían no sólo determinar la cantidad de flujo salival, sino también, el padecimiento de xerostomía, debido a que la afectación del parénquima glandular descrito en ambas enfermedades, desintegraría la mucina salival, que permite el estado de permanente humectación oral(5). De este modo, independiente de la cantidad de saliva producida, un sujeto podría relatar xerostomía. Por este motivo, se hace necesario no solo cuantificar las proteínas en saliva, sino también caracterizarlas, ya que podrían estar determinando la sensación de humectación bucal ${ }^{(24,25)}$.

Existe evidencia que establece una relación entre mayor consumo de fármacos e hiposialia ${ }^{(4,26)}$. En nuestro estudio, si bien la diferencia no fue significativa, el consumo de medicamentos fue mayor en el grupo de sujetos xerostómicos no hiposiálicos, lo que podría sugerir un efecto farmacológico a nivel central que no involucre necesariamente, menor producción salival. Sería interesante para evaluar este aspecto, no sólo ampliar la muestra para establecer si esta diferencia es significativa, sino que también, estudiar variables psicológicas, niveles de estrés y ansiedad en los sujetos afectados.

Finalmente, si bien en nuestro estudio, no fue posible establecer diferencias en las características cualitativas de $\mathrm{pH}$ y proteínas en saliva entre sujetos xerostómicos con y sin hiposialia, detectamos que enfermedades con carácter autoinmune como Artritis Reumatoide y Síndrome de Sjögren tienen mayor frecuencia en sujetos con xerostomía con hiposialia, lo que apoya el hecho que el síntoma de boca seca tiene una etiología multifactorial, en la que también podrán interactuar fenómenos sicológicos y neuropáticos, que necesariamente deben ser evaluados para mejorar las terapias que buscan disminuir el síntoma.

Financiamiento: FONIS SA12I2207 y fellowship OAS-GCUB.

\section{Bibliografía}

1. Niklander S, Veas L, Barrera C, Fuentes F, Chiappini G, Marshall M. Risk factors, hyposalivation and impact of xerostomia on oral health-related quality of life. Braz Oral Res. 2017;31(14):1-9.

2. Atkinson JC, Baum BJ. Salivary enhancement: current status and future therapies. J Dent Educ. 2001;65(10):1096-1101.

3. Liu B, Dion MR, Jurasic MM, Gibson G, Jones JA. Xerostomia and salivary hypofunction in vulnerable elders: prevalence and etiology. Oral Surg Oral Med Oral Pathol Oral Radiol. 2012;114(1):52-60.

4. Porter SR, Scully C. Adverse drug reactions in the mouth. Clin Dermatol. 2000;18(5):525-532.

5. Navazesh M, Mulligan RA, Kipnis V, Denny PA, Denny PC. Comparison of whole saliva flow rates and mucin concentrations in healthy Caucasian young and aged adults. J Dent Res. 1992;71(6):1275-1278.

6. Kossioni AE, Karkazis HC. Socio-medical condition and oral functional status in an older institutionalised population. Gerodontology. 1999;16(1):21-28.

7.Wolff, A., Joshi, R. K., Ekström, J., Aframian, D., Pedersen, A. M. L., Proctor, G., et al. A Guide to medications inducing salivary gland dysfunction, xerostomia, and subjective sialorrhea: A systematic review sponsored by the World Workshop on Oral Medicine VI. Drugs R D. 2016;27(3):505

8. Fox PC, Busch KA, Baum BJ. Subjective reports of xerostomia and objective measures of salivary gland performance. J Am Dent Assoc. 1987;115(4):581-584.

9. Wilson CB. An updated Declaration of Helsinki will provide more protection. Nat Med. 2013;19(6):664

10. Márton K, Madléna M, Bánóczy J, Varga G, Fejérdy P, Sreebny LM, et al. Unstimulated whole saliva flow rate in relation to sicca symptoms in Hungary. Ora Dis. 2008;14(5):472-477.

11. Silvestre-Donat FJ, Miralles-Jordá L, Martínez-Mini V. Protocol for the clinical management of dry mouth. Med Oral. 2004;9(4):273-279.

12. Bardow A, Nyvad B, Nauntofte B. Relationships between medication intake, complaints of dry mouth, salivary flow rate and composition, and the rate of tooth demineralization in situ. Arch Oral Biol. 2001;46(5):413-423.

13. Lukacs JR, Largaespada LL. Explaining sex differences in dental caries prevalence: saliva, hormones, and "life-history" etiologies. Am J Hum Biol. 2006;18(4):540-555.

14. Mirzaii-Dizgah I, Agha-Hosseini F. Unstimulated whole saliva parathyroid hormone in postmenopausal women with xerostomia. J Contemp Dent Pract. 2011;12(3):196-199.
15. Bergdahl M, Bergdahl J. Low unstimulated salivary flow and subjective oral dryness: association with medication, anxiety, depression, and stress. J Dent Res. 2000;79(9):1652-1658

16. Fenoll-Palomares C, Muñoz Montagud JV, Sanchiz V, Herreros B, Hernández V, Mínguez $\mathrm{M}$, et al. Unstimulated salivary flow rate, $\mathrm{pH}$ and buffer capacity of saliva in healthy volunteers. Rev Esp Enferm Dig. 2004;96(11):773-783.

17. Chimenos-Kustner E, Marques-Soares MS. Burning mouth and saliva. Med Oral. 2002;7(4):244-253.

18. Valicena M, Escalona LA. Manejo Terapeútico del paciente con Xerostomía Acta Odontológica Venezolana. 2001;39(1):70-79

19. Schipper RG, Silletti E, Vingerhoeds MH. Saliva as research material: biochemical, physicochemical and practical aspects. Arch Oral Biol. 2007;52(12):1114-1135.

20. Messana I, Cabras T, Inzitari R, Lupi A, Zuppi C, Olmi C et al. Characterization of the human salivary basic proline-rich protein complex by a proteomic approach. J Proteome Res. 2004;3(4):792-800.

21. Field EA, Longman LP, Bucknall R, Kaye SB, Higham SM, Edgar WM. The establishment of a xerostomia

clinic: a prospective study. Br J Oral Maxillofac Surg. 1997;35(2):96-103

22. Vitali C. Classification criteria for Sjögren's syndrome. Ann Rheum Dis. 2003;62(1):94-95.

23. Aitken-Saavedra J, Rojas-Alcayaga G, Maturana-Ramírez A, Escobar-Álvarez A, Cortes-Coloma A, Reyes- Rojas M, et al. Salivary gland dysfunction markers in type 2 diabetes mellitus patients. J Clin Exp Dent. 2015;7(4):501-505.

24. Davies AN, Singer J. A comparison of artificial saliva and pilocarpine in radiationinduced xerostomia. J Laryngol Otol. 1994;108(8):663-665.

25. Laine M, Pienihäkkinen K, Leimola-Virtanen R. The effect of repeated sampling on paraffin-stimulated salivary flow rates in menopausal women. Arch Oral Biol. 1999;44(1):93-95.

26. Pajukoski H, Meurman JH, Halonen P, Sulkava R. Prevalence of subjective dry mouth and burning mouth in hospitalized elderly patients and outpatients in relation to saliva, medication, and systemic diseases. Oral Surg Oral Med Oral Pathol Oral Radiol Endod. 2001;92(6):641-649. 